\title{
A performance Evaluation of Transform Domain Algorithm in Watermarking Based on Different Levels of Sub-Bands of Discrete Wavelet Transform
}

\author{
Musbah J. Aqel ${ }^{1}$, Aliyu Umar ${ }^{2}$, Mary Agoyi ${ }^{3}$ \\ ${ }^{1,2,3}$ Department of Management Information Systems, Cyprus Internatioan University, Lefkosa, North Cyprus
}

\section{Article Info}

Received Jun 9, 2019

\section{Keyword:}

Wavlet transform

Watermarking

Singular value decompestion

\begin{abstract}
This study proposed to evaluate the performance of transform domain algorithms in watermarking based on different level of sub bands of discrete wavelet transform (DWT) that can be used to overcome illegal copy, manipulation, distribution and reproduction of image on the network by implementing watermarking algorithm based on different levels that is lowlevel (LL), low- high (LH), high-low (HL), High-high (HH) and singular value decomposition (SVD) on image for their robustness, and imperceptibility. Moreover, two parameters were applied to evaluate the performance. That is, peak signal to noise ratio (PSNR) is used to measure imperceptibility of the digital image while normalize cross correlation (NCC) is applied to measure the robustness of digital image. The performance evaluations of the proposed method were implemented in MATLAB. The results showed that the described method imperceptible of HL level sub-bands than other levels of sub-bands. While the robustness is achieved by all levels sub-bands.
\end{abstract}

\section{Corresponding Author:}

First author, Musbah J. Aqel

Cyprus Internataional University

Lefkosa, North Cyprus

maqel@ciu.edu.tr

\section{Introduction}

With the new progress in the internet technology, it makes digital contents to be accessible by various users. However, this technology has advantages such as digital content can be access easily with less time, furthermore, digitalization also has disadvantages like illegal copy, distribution, reproduction and manipulation on digital contents without the knowledge of the owners [14]. These problems can be solved by applying watermark. Digital watermarking is a powerful scheme applied to covered data in order to protect illegal copy, reproduction and security issues of digital content like image, audio and text. Digital watermarking is done by applying strong, specific and applicable techniques which play a vital role in digital watermarking [1], [7].

Recently, digital watermarking has showed one of the crucial research aspects in the area of signal processing and information security. The aim is to insert un-removable digital watermarking into original content and also the same watermark can be extracted to solve copyright protection. Therefore, the inserted digital watermark must have the following characteristic; Imperceptibility and robustness [16].

Maximum of information; an embedded watermark data should have capacity to contain largest watermark information [10]. However, maximum of information indicates the amount of information that can be embedded into multimedia contents, capable of hiding watermarks that can be detected for the authentication and copyright protection based on condition of imperceptibility and robustness. However, embedding large watermark information it can be more difficult work in digital image watermarking. Maximum of information is the number of inserted bits into pixel of the digital image. 


\subsection{Related work}

Several studies have been carried out in the field of watermarking. Some of them are as follow:

Kaur et..al., developed a digital watermarking algorithm that based on DWT, FFT, Arnold transform and SVD. Watermarking embedding process is done by applying cover image on FFT, then applied DWT on image to decompose it into four sub-bands and then, applied SVD. The developed method implemented in MATLAB. PSNR is applied for imperceptibility and the obtained results proved that the developed technique is imperceptible [6].

Laura et al. proposed a digital image watermarking technique based on DWT, Chirp Z-transform, entropy and SVD. These techniques embed the digital watermark in colored image. The obtained results of the developed algorithm showed that is robustness and imperceptiblity against various signal operation attack like compression and histogram equalization [4].

Gunjal\& Mali [2] proposed a watermarking scheme. This scheme is developed by using Java.net and MATLAB. The proposed scheme is based on DWT- Fast wavelet Hadamard transform. (DWT-FWHT-SVD) and DWT domains. However, orthogonal wavelet and symmetric is adopted for DWT decomposition and also Fiboacci-Lucast transform together with affine transforms that are applied for inserting security. The experimental result shows the good performance in DWT-FWHT-SVD domain than DWT domain in terms of security and robustness [2].

Rassem et al., described digital watermarked algorithms based on DWT -SVD and Redundant DWT -SVD, RDWTSVD. These algorithms are to evaluate their performance based on robustness and imperceptibility. The obtained results proved that the proposed techniques are both robust against various attacks, but DWT-SVD it resists more attacks than RDWT-SVD such as scaling and filtering, JPEG compression [11].

Theakur, proposed digital watermarking algorithms based on the DWT, FFT and SVD for imperceptibility and robustness on digital image watermarking. The proposed method is implemented in MATLAB. The embedding process of this technique is done in such a way that, watermark signal can be embedded without affecting the quality of the original image. The proposed algorithm showed that is robust and imperceptible based on the experimental results [15].

Saxena et al, developed a watermarking technique based on DWT, DCT and SVD with semi-blind algorithm has been applied. The performance evaluation of the proposed technique is done by performing PSNR and CC for both imperceptibility and robustness. Various attacks have been applied in MATLAB to measure the robustness of the proposed method such as rotation, cropping, translation and noise. However, the obtained result showed that the propose algorithms is robust against several attacks and also imperceptible [13].

Pandurangan and logashanmugamproposed watermarking techniques by applying DWT-DCT with SVD transform on digital image. In inserting process, the DCT is carried out on the original image by using zigzag sequence, and then represent the DCT coefficient into four quadrants. Furthermore, SVD is used to each quadrant. The experimental result showed that there is good robustness and transparency on the proposed algorithm [9].

Savakar\&Ghuli, proposed a watermarking technique $\mathrm{m}$ based on DWT- FFT on digital image. DWT is carried out on original image for watermarking embedding process. Then, FFT is applied to convert input signal from spatial domain format to a frequency domain format. The evaluation of the proposed techniques is done based on fragility and fidelity, MATLAB 2009a is used as programming tool for simulations. The experimental result demonstrated that, the fragility with use various attacks [12].

Patel \&Nandurbarkar, developed a watermarking technique based on DCT-DWT-SVD, initially watermarking is based on SVD of DC coefficient by implementing a second- level of DWT decomposition and the next method is based on SVD of DCT values of the second-level DWT composition of original image. All these processes are implemented in MATLAB. The experimental results explained that both the two approaches have shown robustness and imperceptiblity against different signal processing [8].

Kang \&Iwamura, proposed digital image watermarking technique using various frequency coefficients that derived from DCT values of the second level DWT transform. Bose-Chaudhuri-Hocquen $(\mathrm{BCH})$ code is used for watermark and $\mathrm{BCH}$ code is applied for synchronous data. The proposed technique is used for statistical analysis to choose the best position in the frequency domain for watermark embeds [5].

Mardolkar\&Shenvi, proposed a blind digital watermarking technique using DWT and DCT transform. Firstly, the original image was decomposed by 2 levels DWT, then Block DCT is used to the selected DWT sub-band. However, watermark inserting process is done by carried the low frequency coefficient of every selected DCT. The watermarked 
original image is applied to several watermark attacks. The obtained results demonstrated that the developed scheme has shown robustness [3].

\section{Proposed method}

The proposed method consists of inserting and extracting watermark on digital on digital contents. Digital watermarking algorithm applied on this research is DWT-SVD based on resolution of different sub-bands level that is LL, LH, HL and $\mathrm{HH}$ on image to protect multimedia content from illegal copy, manipulation, reproduction and distribution of digital content over the internet. The digital images used for inserting watermark are; the host image used is House and the watermark applied is signature.

In this research, the scheme developed by applied MATLAB 2015. The two grey images used in this method are House for original image while Signature for watermark image.

\section{Process of Embedding image watermarking based on LL}

The DWT-SVD digital watermark embedding technique explained below:

1. Apply Discrete Wavelet Transform in order to decompose the original image B into LL of one sub- band. As shown in the following equation.

$$
[\mathrm{LL}, \mathrm{LH}, \mathrm{HL}, \mathrm{HH}]=\mathrm{DWT}_{2}(\mathrm{~B})
$$

2. Apply SVD to LL of one level sub-band image $\mathrm{K}$ as given in this equation $[U S V]=S V D(K)$

3. Apply SVD to the watermark image $\mathrm{C}$ as given in this equation

$$
\left[\mathrm{U}_{1} \mathrm{~S}_{1} \mathrm{~V}_{1}\right]=\operatorname{SVD}(\mathrm{C})
$$

4. The decomposed cover image that used SVD watermark with scaling factor. as given below

$$
\mathrm{S}_{2}=\mathrm{S}+\alpha \mathrm{S}_{1}
$$

5. The orthogonal matrix of the cover image is added along with SVD $\mathrm{S}_{2}$ to that is watermarked.

The equation is given below

$\mathrm{LL} 2=\mathrm{U} * \mathrm{~S}_{2} * \mathrm{~V}$,

The inverse of the DWT as shown in this equation

$\mathrm{D}=\mathrm{IDWT}_{2}\left[\mathrm{LL}_{2} \mathrm{LH}_{2} \mathrm{HL}_{2} \mathrm{HH}_{2}\right]$

\section{Process of Extracting Image Watermarking Based on DWT-SVD (LL)}

Perform one-level wavelet transform on the watermarked image $\mathrm{D}$, as shown below

$$
\left[\mathrm{LL}_{3} \mathrm{LH}_{3} \mathrm{HL}_{3} \mathrm{HH}_{3}\right]=\mathrm{DWT}_{2}(\mathrm{D})
$$

Perform SVD based on the selected $\mathrm{LL}_{3}$ sub-bands of the watermarked image $\mathrm{N}$ as shown below

$\left[\mathrm{U}_{3} \mathrm{~S}_{3} \mathrm{~V}_{3}\right]=\mathrm{SVD}(\mathrm{N})$

Perform one-level wavelet transform on the original image $\mathrm{B}$ as show below

$\left[\mathrm{LL}_{4} \mathrm{LH}_{4} \mathrm{HL}_{4} \mathrm{HH}_{4}\right]=\mathrm{DWT}_{2}(\mathrm{~B})$

Perform SVD on the $\mathrm{LL}_{4}$ sub-bands of the original image as given below

$\left[\mathrm{U}_{4} \mathrm{~S}_{4} \mathrm{~V}_{4}\right]=\operatorname{SVD}(\mathrm{K})$

The decomposed watermark image and the decomposed original image that used singular value can be divided by scaling factor as given in below equation

$\mathrm{S}_{5}=\left(\mathrm{S}_{3}-\mathrm{S}_{4}\right) / \alpha$

Perform SVD on the watermark image $\mathrm{Q}$ as given in this equation below

$\left[\mathrm{U}_{6} \mathrm{~S}_{6} \mathrm{~V}_{6}\right]=\mathrm{SVD}(\mathrm{P})$

The watermark can be obtain as shown in this equation below

$$
\mathrm{YY}=\mathrm{U}_{6} * \mathrm{~S}_{5} * \mathrm{~V}_{6}{ }^{\prime} \quad(3.13)
$$

\section{Process of Embedding image watermarking based on LH}

The DWT-SVD digital watermark embedding technique explained below:

Apply DWT to decompose the original image B into LH of one sub- band. As shown in the following equation.

$[\mathrm{LL}, \mathrm{LH}, \mathrm{HL}, \mathrm{HH}]=\mathrm{DWT}_{2}(\mathrm{~B})$

Apply SVD to LH of one level sub-band image $\mathrm{K}$ as given in this equation $[U S V]=S V D(K)$ (315) 
Apply SVD to the watermarked image (C) as given in this equation

$\left[\mathrm{U}_{1} \mathrm{~S}_{1} \mathrm{~V}_{1}\right]=\mathrm{SVD}(\mathrm{C})$

The decomposed cover image that used SVD watermarked with scaling factor as given below

$\mathrm{S}_{2}=\mathrm{S}+\alpha \mathrm{S}_{1} \quad$ (3.17)

The orthogonal matrix of the cover image is added along with SVD $\mathrm{S}_{2}$ to that is watermarked

The equation is given below

$\mathrm{LH} 2=\mathrm{U} * \mathrm{~S}_{2} * \mathrm{~V}$,

The inverse of the DWT as shown in this equation

$\mathrm{D}=\mathrm{IDWT}_{2}\left[\mathrm{LL}_{2} \mathrm{LH}_{2} \mathrm{HL}_{2} \mathrm{HH}_{2}\right]$

\section{Process of Extracting Image Watermarking Based on DWT-SVD (LH)}

Perform one-level wavelet transform on the watermarked image $\mathrm{D}$, as shown below

$$
\left[\mathrm{LL}_{3} \mathrm{LH}_{3} \mathrm{HL}_{3} \mathrm{HH}_{3}\right]=\mathrm{DWT}_{2} \text { (D) }
$$

Perform SVD based on selected $\mathrm{LL}_{3}$ sub-bands of the watermarked image $\mathrm{N}$ as shown below

$\left[\mathrm{U}_{3} \mathrm{~S}_{3} \mathrm{~V}_{3}\right]=\mathrm{SVD}(\mathrm{N})$

Perform one-level wavelet transform on the original image $\mathrm{B}$ as show below

$\left[\mathrm{LL}_{4} \mathrm{LH}_{4} \mathrm{HL}_{4} \mathrm{HH}_{4}\right]=\mathrm{DWT}_{2}$ (B)

Perform SVD on the $\mathrm{LH}_{4}$ sub-bands of the original image as given below

$\left[\mathrm{U}_{4} \mathrm{~S}_{4} \mathrm{~V}_{4}\right]=\mathrm{SVD}(\mathrm{K})$

The decomposed watermark image and the decomposed original image that used singular value can be divided by scaling factor as given in below equation

$\mathrm{S}_{5}=\left(\mathrm{S}_{3}-\mathrm{S}_{4}\right) / \alpha \quad(3.24)$

Perform SVD on the watermark image $\mathrm{Q}$ as given in this equation below

$\left[\mathrm{U}_{6} \mathrm{~S}_{6} \mathrm{~V}_{6}\right]=\mathrm{SVD}(\mathrm{P})$

The watermark can be obtain as shown in this equation below

$$
\mathrm{YY}=\mathrm{U}_{6} * \mathrm{~S}_{5} * \mathrm{~V}_{6}^{\prime} \quad(3.26)
$$

Process of Embedding image watermarking based on HL

The DWT-SVD digital watermark embedding technique explained below:

Apply Discrete Wavelet Transform to decompose the original image B into HL of one sub- band. As shown in the following equation.

$[\mathrm{LL}, \mathrm{LH}, \mathrm{HL}, \mathrm{HH}]=\mathrm{DWT}_{2}(\mathrm{~B})$

Apply SVD to $\mathrm{HL}$ of three level sub-band image $\mathrm{K}$ as given in this equation $[U S V]=\operatorname{SVD}(K)$

(3.28)

Apply SVD to the watermark image $(\odot)$ as given in this equation

$\left[\mathrm{U}_{1} \mathrm{~S}_{1} \mathrm{~V}_{1}\right]=\mathrm{SVD}(\mathrm{C})$

The decomposed cover image that used SVD watermark with scaling factor as given below

$\mathrm{S}_{2}=\mathrm{S}+\alpha \mathrm{S}_{1} \quad$ (3.30)

The orthogonal matrix of the cover image is added along with $\mathrm{SVD} \mathrm{S}_{2}$ to that is watermarked

The equation is given below

$\mathrm{HL} 2=\mathrm{U} * \mathrm{~S}_{2} * \mathrm{~V}$,

The inverse of the DWT as shown in this equation

$\mathrm{D}=\mathrm{IDWT}_{2}\left[\mathrm{LL}_{2} \mathrm{LH}_{2} \mathrm{HL}_{2} \mathrm{HH}_{2}\right]$

Process of Extracting Image Watermarking Based on DWT-SVD (HL)

Perform one-level wavelet transform on the watermarked image $\mathrm{D}$, as shown below
$\left[\mathrm{LL}_{3} \mathrm{LH}_{3} \mathrm{HL}_{3} \mathrm{HH}_{3}\right]=\mathrm{DWT}_{2}(\mathrm{D})$
(3.33)

Perform SVD based on selected $\mathrm{HL}_{3}$ sub-bands of the watermarked image $\mathrm{N}$ as shown below

$\left[\mathrm{U}_{3} \mathrm{~S}_{3} \mathrm{~V}_{3}\right]=\mathrm{SVD}(\mathrm{N})$

Perform three-level wavelet transform on the original image $\mathrm{B}$ as show below

$\left[\mathrm{LL}_{4} \mathrm{LH}_{4} \mathrm{HL}_{4} \mathrm{HH}_{4}\right]=\mathrm{DWT}_{2}(\mathrm{~B})$

Perform SVD on the $\mathrm{HL}_{4}$ sub-bands of the original image as given below

$\left.\mathrm{U}_{4} \mathrm{~S}_{4} \mathrm{~V}_{4}\right]=\mathrm{SVD}(\mathrm{K})$

The decomposed watermark image and the decomposed original image that used singular value can be divided by scaling factor as given in below equation

$\mathrm{S}_{5}=\left(\mathrm{S}_{3}-\mathrm{S}_{4}\right) / \alpha \quad(3.37)$

Perform SVD on the watermark image $\mathrm{Q}$ as given in this equation below 
$\left[\mathrm{U}_{6} \mathrm{~S}_{6} \mathrm{~V}_{6}\right]=\mathrm{SVD}(\mathrm{P})$

The watermark can be obtain as shown in this equation below $\mathrm{YY}=\mathrm{U}_{6} * \mathrm{~S}_{5} * \mathrm{~V}_{6}^{\prime} \quad(3.39)$

\section{Process of Embedding image watermarking based on $\mathrm{HH}$}

The DWT-SVD digital watermark embedding technique explained below:

Apply DWT to decompose the original image B into HH of one sub- band. As shown in the following equation.

$[\mathrm{LL}, \mathrm{LH}, \mathrm{HL}, \mathrm{HH}]=\mathrm{DWT}_{2}(\mathrm{~B})$

(3.40)

Apply SVD to $\mathrm{HH}$ of three level sub-band image $\mathrm{K}$ as given in this equation $[U S V]=\operatorname{SVD}(K)$ (3.41)

Apply SVD to the watermarked image (C) as shown in the following equation
$\left[\mathrm{U}_{1} \mathrm{~S}_{1} \mathrm{~V}_{1}\right]=\mathrm{SVD}(\mathrm{C})$
(3.42)

The decomposed cover image that used singular value of decomposed watermark with scaling factor as given below

$\mathrm{S}_{2}=\mathrm{S}+\alpha \mathrm{S}_{1} \quad$ (3.43)

The orthogonal matrix of the cover image is added along with SVD $S_{2}$ to that is watermarked

The equation is given below

$\mathrm{HL} 2=\mathrm{U} * \mathrm{~S}_{2} * \mathrm{~V}$

The inverse of the DWT as shown in this equation

$\mathrm{D}=\mathrm{IDWT}_{2}\left[\mathrm{LL}_{2} \mathrm{LH}_{2} \mathrm{HL}_{2} \mathrm{HH}_{2}\right]$

\section{Process of Extracting Image Watermarking Based on DWT-SVD}

Perform four-level wavelet transform on the watermarked image $\mathrm{D}$, as shown below
$\left[\mathrm{LL}_{3} \mathrm{LH}_{3} \mathrm{HL}_{3} \mathrm{HH}_{3}\right]=\mathrm{DWT}_{2}(\mathrm{D})$

Perform SVD based on selected $\mathrm{HH}_{3}$ sub-bands of the watermarked image $\mathrm{N}$ as shown below

$\left[\mathrm{U}_{3} \mathrm{~S}_{3} \mathrm{~V}_{3}\right]=\mathrm{SVD}(\mathrm{N})$

Perform one-level wavelet transform on the original image $\mathrm{B}$ as show below
$\left[\mathrm{LL}_{4} \mathrm{LH}_{4} \mathrm{HL}_{4} \mathrm{HH}_{4}\right]=\mathrm{DWT}_{2}(\mathrm{~B})$
(3.48) Perform SVD on the $\mathrm{HH}_{4}$ sub-bands of the

original image as given below

$\left[\mathrm{U}_{4} \mathrm{~S}_{4} \mathrm{~V}_{4}\right]=\mathrm{SVD}(\mathrm{K})$

The decomposed watermark image and the decomposed original image that used singular value can be divided by scaling factor as given in below equation

$\mathrm{S}_{5}=\left(\mathrm{S}_{3}-\mathrm{S}_{4}\right) / \alpha \quad(3.50)$

Perform SVD on the watermark image $\mathrm{Q}$ as given in this equation below

$\left[\mathrm{U}_{6} \mathrm{~S}_{6} \mathrm{~V}_{6}\right]=\mathrm{SVD}(\mathrm{P})$

The watermark can be obtain as shown in this equation below

$\mathrm{YY}=\mathrm{U}_{6} * \mathrm{~S}_{5} * \mathrm{~V}_{6}{ }^{\prime}(3.52)$

\section{Performance analysis}

This chapter evaluates the performance of the described technique, experimental result and lastly observations. This scheme is implemented in MATLAB 2015. The algorithm proposed is based on digital Image watermarking. Therefore, the original image used on this technique is House image while watermark data is signature. After the watermarked data the experimental result showed that, between the host images, and watermarked are identical. The PSNR is applied on watermarked image Therefore the PSNR is measured for all the four level of sub band of DWT techniques. That is, LL, LH, HL and HH the proposed scheme showed that PSNR has the higher values it means that the proposed method is imperceptible. Many attacks were applied on image to evaluate the robustness of the proposed method. NCC is used to evaluate robustness of digital image.

\section{Experimental Result}

The described scheme is analyzed by using robustness and imperceptibility. The imperceptibility is to show the qualities of the watermarked image. That is, the present of inserted watermark image could not make any changes on the originalimage. The robustness is used to evaluate the resistance of digital image watermark against various attacks and signal operations on images like salt and pepper, Gaussian noise, rotation, cropping,and scaling. 


\section{Imperceptibility Testing for Image}

To evaluate imperceptibility of digital image watermarking, PSNR is applied on digital images

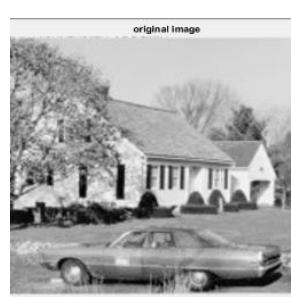

Original image (LL)

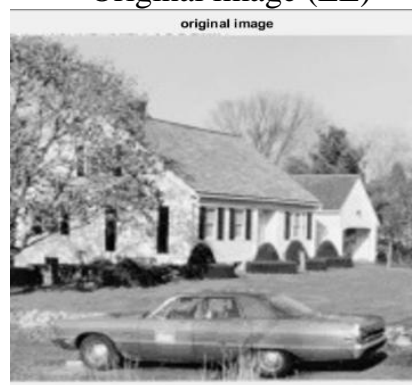

Original image (HL)

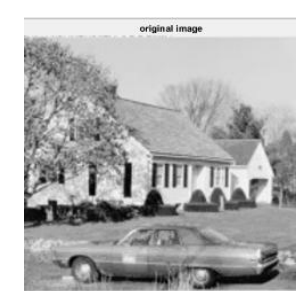

Original image (LH)

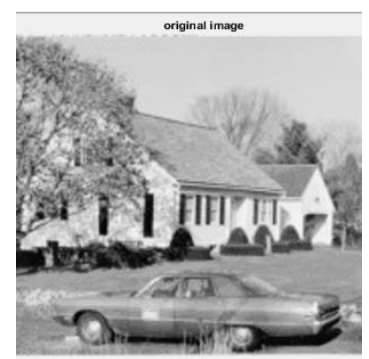

Original image $(\mathrm{HH})$

Figure (1) shown all original images above

Figure (1) has shown the entire original image used for different levels of sub-band of DWT based on LL, LH, HL and $\mathrm{HH}$ for digital watermarking.

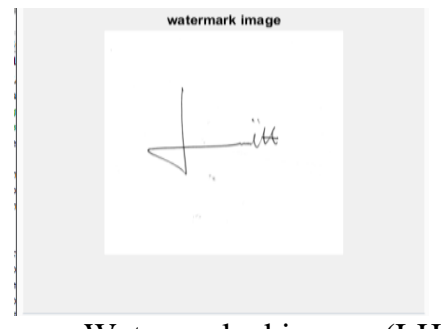

Watermarked image (LH)

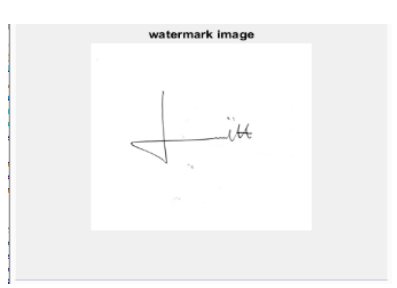

watermarked image (LL)

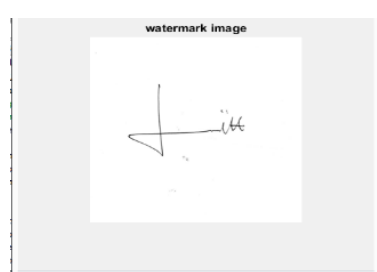

Watermarked image (HL) Figure (2) all watermark image

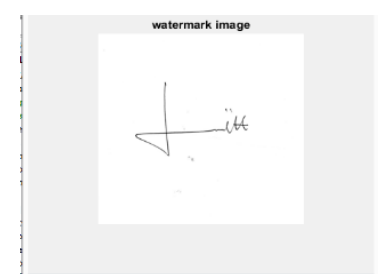

Watermarked image $(\mathrm{HH})$

Figure (2) has shown the embedding signature used for differetent levels of sub-band of DWT based on LL, LH, HL, and $\mathrm{HH}$ into the original image as watermarked images.

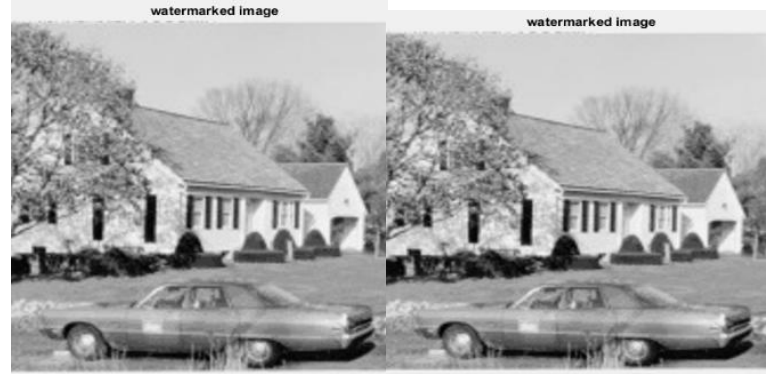

Watermarking image (LL) Watermarking image (LH) 
$($ PSNR $=39.5580)$

$(\mathrm{PSNR}=39.5973)$

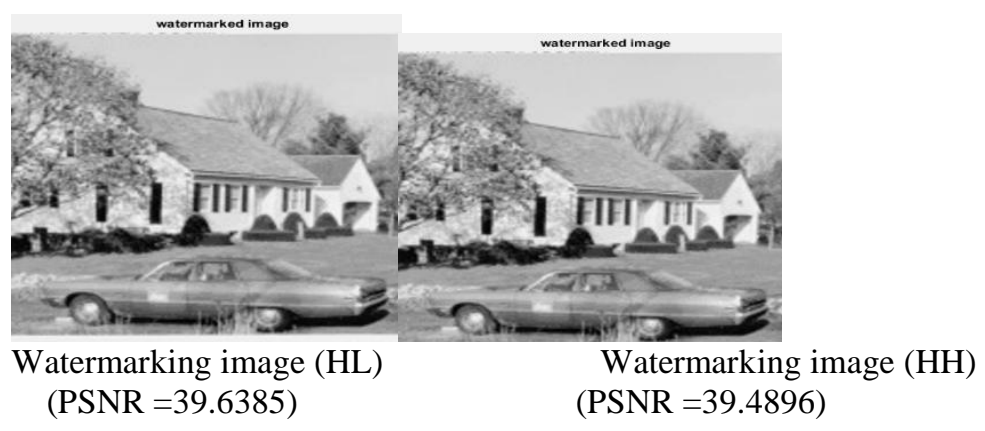

Figure (3) all watermarked images above

Figure 3 above shown the entire watermarked image used for different levels of sub-band of DWT based on LL, LH, HL and $\mathrm{HH}$ in which signature is used as watermark data,

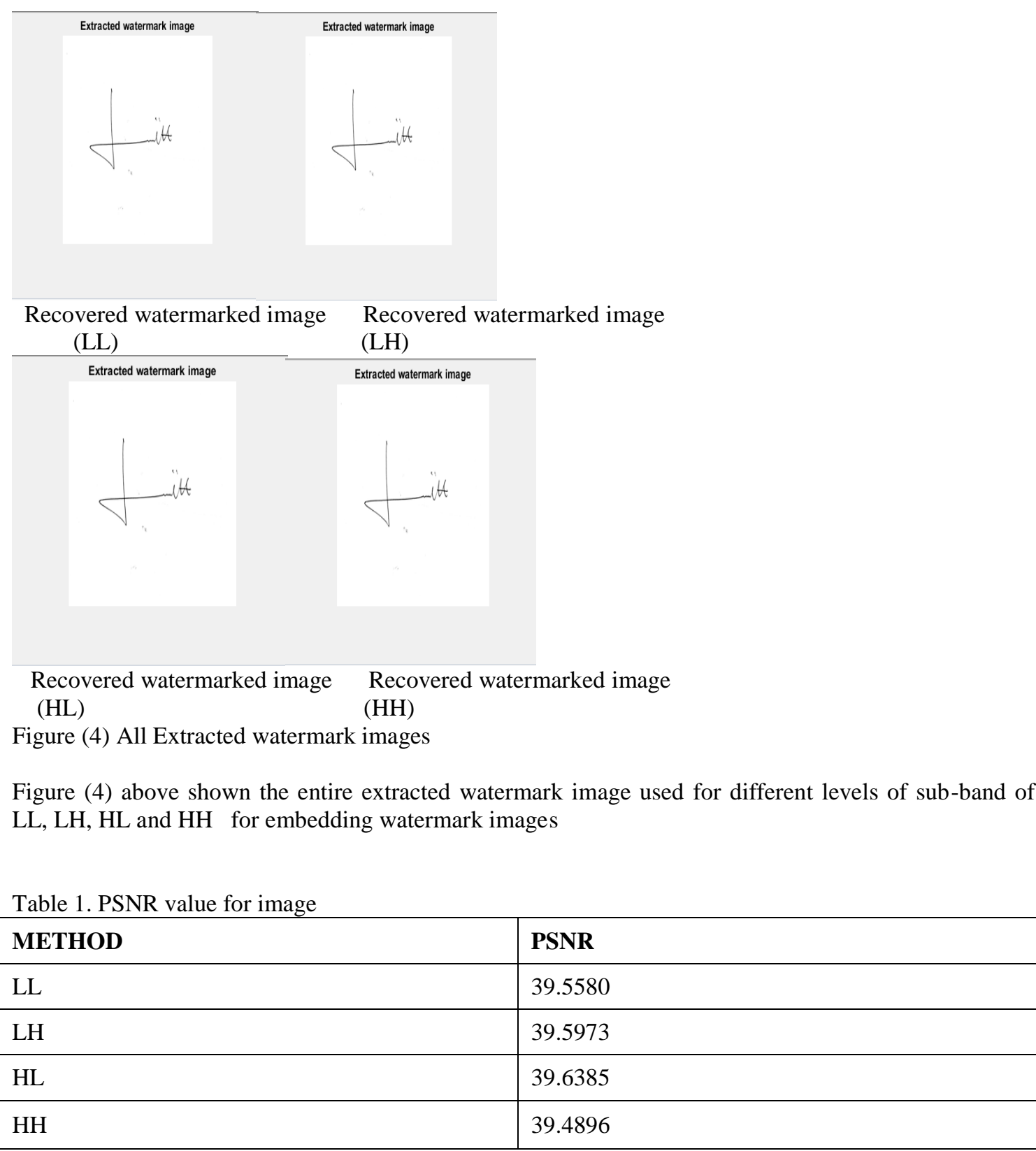


Table 2. PSNR of attack (image)

\begin{tabular}{|l|l|l|l|l|}
\hline Attacks & LL & LH & HL & HH \\
\hline Filtering & 30.9856 & 31.3933 & 31.4058 & 31.4164 \\
\hline Scaling & 37.2303 & 40.7783 & 40.8052 & 40.7969 \\
\hline Rotation & 8.9283 & 9.0266 & 9.1545 & 9.0109 \\
\hline Flipping & 13.8043 & 13.8566 & 13.8846 & 13.7488 \\
\hline Blurring & 24.2394 & 24.2508 & 24.2593 & 24.2418 \\
\hline Contrast & 23.6683 & 23.5475 & 23.5130 & 23.1509 \\
\hline Cropping & 10.8272 & 10.8657 & 10.8697 & 10.7611 \\
\hline Gaussian noise & 20.1019 & 20.0562 & 20.0919 & 20.0751 \\
\hline Hit-equal & 14.4102 & 14.3554 & 14.3649 & 14.3777 \\
\hline Gamma corr & 9.7166 & 9.7162 & 9.7137 & 9.7109 \\
\hline Salt and Pepper & 25.0648 & 25.1301 & 25.0770 & 25.2726 \\
\hline Sharpening & 30.5841 & 26.9869 & 28.5897 & 30.5399 \\
\hline
\end{tabular}

Table2 explained the PSNR values of watermarked image based on different levels of sub-bands of DWT. However, different attacks were used to test the imperceptibly of watermarking image.

\section{Robustness Testing for Image}

To evaluate robustness of digital watermarking, NCC is used. The NCC is applied on digital image. In the field of watermarking the range of NCC is between 1.0 and -1.0. If the numbers of NCC is exactly one. It means there is a perfect identity between the original watermarked image and recovered watermarks

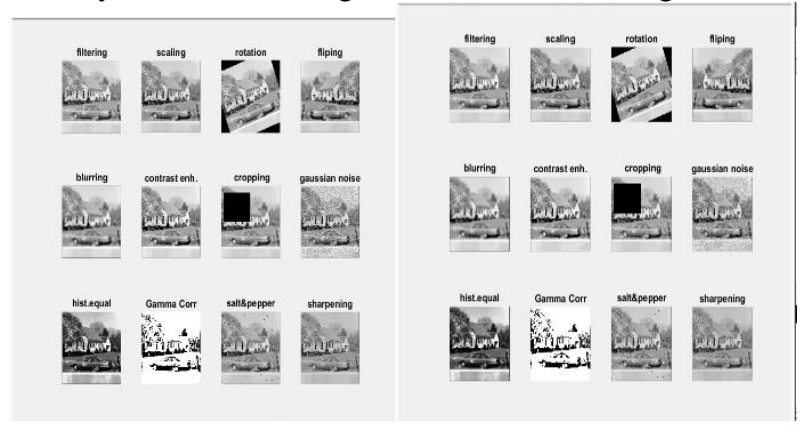

Figure 5. DWT-SVD (11) attacks for NCC

Figure (5) watermarked images are used together with various attack to measure NCC of the watermarked data based on LL sub-bands of DWT.

Table 4.3 NCC value for image attacks

\begin{tabular}{|l|l|l|l|l|}
\hline Attacks & LL & LH & HL & HH \\
\hline Filtering & 0.7733 & 0.7761 & 0.7687 & -0.8009 \\
\hline Scaling & 0.6711 & 0.6714 & 0.6704 & 0.6687 \\
\hline Rotation & -0.5957 & -0.5582 & -0.5940 & -0.6143 \\
\hline Flipping & 0.1663 & 0.3696 & 0.1341 & 1.0000 \\
\hline Blurring & -0.7138 & -0.7138 & -0.7136 & -0.7141 \\
\hline Contrast & 0.7203 & 0.7194 & 0.7190 & 0.8252 \\
\hline Cropping & -0.7088 & -0.7089 & -0.7090 & 0.3721 \\
\hline Gaussian noise & 0.5603 & 0.5573 & 0.5593 & 0.5598 \\
\hline Hit-equal & 0.6512 & 0.6485 & 0.6636 & 0.8802 \\
\hline Gamma corr & 0.6759 & 0.6736 & 0.6774 & 0.7351 \\
\hline Salt and Pepper & 0.5702 & 0.5728 & 0.5720 & 0.5793 \\
\hline Sharpening & 0.7426 & 0.7416 & 0.7422 & 0.8597 \\
\hline
\end{tabular}

Table 4.3 explained the NCC values of watermarked image based on different levels of sub-bands of DWT. However, many attacks were used to test the robustness of watermarked image. 


\section{Conclusion}

With rapid development in internet technologies, it makes many users to access digital content easily. The digital data can be exchange over the internet and users can copy digital data, produced it without the knowledge of the ownership. Therefore, watermarking is applied to solve the issues of illegal copy, production, copyright protection and signal operation. In this study, we proposed a Performance Evaluation of Transform Algorithm in Watermarking Domain based on different Levels of sub-bands of DWT. The explained the results showed that the described method is imperceptible on HL level sub-bands than LL sub-band, LH sub-bands and the HH sub-bands. While for the robustness almost all the four levels of sub-bands achieved the robustness because based the on-approximation method most of the value of CC is equal to one.

\section{References}

[1] Chowdhury,A., Shahjamal, M. \& Biswas, K. (2014) A New DWT-SVD Based Image Watermarking Technique By Utilizing The Features Human Visual System, International Journal of Research in Computer Engineering and Electronics. ISSN (3)6, 11-15

[2] Gunjal, B.L, Mali, S.N.(2014). Comparative Performance Analysis of Digital Image Watermarking Scheme in DWT and DWT-FWHT-SVD Domains.2014 Annual IEEE India Conference (INDICON).

[3] Mardolkar, S.B \&Shenvi, N. (2016). A Blind digital Watermarking Algorithm based on DWT-DCT Transformation, International Journal of Innovative Research in Electrical, Electronics, Instrumentation and Control Engineering Nitte Conference on Advances in Electrical Engineering NCAEE-2016, DOI 10.17148, (3)2 ,212-216.

[4] Laura, L.Rasti, P.Agoyi, M.\&Anbarjafari, G. (2015) .A Robust Color Image Watermarking Scheme Using Entropy and QR Decomposion,(24)4, 1-8, DOI: 10.13164/re.2015.1025.

[5] Kang, H.,\&, Iwamura, K.,(2015).Information Hiding Method Using Best DCT and Wavelet Coefficients and Its Watermark Competition, 1218-1235; doi:10.3390/e17031218

[6] Kaur, K.,(2016). Fast Fourier Transform based Hybrid Image Watermarking using Arnold Scrambling, International Journal for Research in Applied Science \& Engineering Technology (IJRASET) (4)2, 204-2013

[7] Laskar, T.A \&Hemachandran, K.(2013). Digital image watermarking techniques and its. Applications. International Journal of Engineering Research \& Technology (IJERT). Vol.2, No.3, pp.1-8

[8] Patel, R., \&Nandurbarkar, A.(2015). Implementation of DCT DWT SVD based watermarking algorithms for copyright protection, (2)2, 340-344

[9] Pandurangan, R. \&Logashanmugam, E. (2014). DCT-SVD And DWT-SVD Image Watermarking Techniques, International Journal of Applied Engineering Research,(9)24, 25833-25839

[10] Potdar, Vidyasagar M. , Han S., Chang E., (2005). A survey of digital image watermarking techniques, $3^{\text {rd }}$ IEEE International Conference on Industrial Informatics (India), pp 709-716.

[11] Rassem, T.H, Makbol, N.M \&Khoo, B.E (2015). Performance Evaluation of RDWT-SVD and DWT-SVD Watermarking schemes, pp.1-9, doi.org/10.1063/1.4965108

[12] Savakar,D.G\&Ghuli, A. (2015).Semi-Fragile Digital Watermarking using Two- Level Discrete Wavelet Transformation and Two-Dimensional Fast Fourier transformation. International Journal of Computer Application, (5) $7.33-42$

[13] Saxena, H., Saxena, P. \&Rastogi, S. ( 2014).DWT-DCT-SVD based semi-blind reference image watermarking scheme using trigonometric function, International Journal of conceptions on Computing and Information Technology, (2)2, 14-18

[14] Shereen Jumaa, Khamis Zidan (2019) Finger vein recognition using parallel enhancement approaches based fuzzy histogram equalization, Periodical of Engineering and Natural Sciences, Vol. 7, No. 1,

[15] Thakur, K (2017) Hybrid DWT, FFT and SVD based Watermarking Techniquefor Different wavelet Transforms.(5)1,7-12

[16] Tao, H. Chongmin, L., Mohamad, Z.J \&, Ahmed N. Abdalla3, A.N (2014) .Robust Image watermarking Theories and Techniques: A Review, Vol.2,pp. 122-138. 